\title{
Histological Evaluation of Osseointegration of 3D Printed Ti6Al4V Screws
}

\author{
Kang-Sik Lee ${ }^{1}$, Hyewon Kim ${ }^{1}$, Eunjeong Choi ${ }^{1}$, Hae-Jin Lee ${ }^{2}$, Byoung-Soo Lee ${ }^{2}$ \\ ${ }^{1}$ Asan Medical Center, University of Ulsan College of Medicine \\ 88 Olympic-ro 43, Songpa-gu, Seoul, Korea \\ knifebrd@hanmail.net; 091608@kist.re.kr; pupet0715@naver.com \\ ${ }^{2}$ Korea Institute of Industrial Technology \\ Gwahakdanji-ro 134-41, Gangneung, Gangwon-do, Korea \\ seajin@kitech.re.kr; leebs@kitech.re.kr
}

\section{Extended Abstract}

Background : Ti6Al4V ELI has been widely used in biomedical devices such as a bone plate, acetabular cups, remoral stems, and heads because of its excellent biocompatibility and good specific strength. Its surface structures including morphologies and irregularities play a crucial role in bone tissue integration, and thus numerous studies have been conducted on chemical and physical surface modification.

Purpose : The aim of this work was to compare the osseointegration of both 3D-printed Ti6Al4V screws with a microrough surface and machined Ti6Al4V screws with a smooth surface using in vivo rabbit animal model.

Methods: The in vivo test specimens were prepared using two kinds of fabrication methods which were 3D-printed manufacturing by an Arcam EBM-A2X device (Arcam AB, Mölndal, Sweden) and machined by a five-axis machine. Two types of surface, micro-rough and smooth, were characterized using micro-computer tomography (micro-CT), scanning electron microscopy (SEM). Two types of screws with different surface structures, micro-rough and smooth, were implanted in bilateral femur condyles of ten New Zealand white rabbits weighing approximately $2.5 \mathrm{~kg}$ (3-4 month old). After two weeks of operation, specimens containing the implanted screws and the area surrounding it were fixed, dehydrated, embedded for undecalcified sectioning, stained with Goldner's trichrome method, and then scanned by a digital slide scanner. To determine osseointegration and bone regeneration around the screw implant, the bone-to-implant contact (BIC) was quantified in interface between bone and implant and histological analysis around screw implant was evaluated.

Results : The new bony tissues contacted with the 3D-printed Ti6Al4V screws were well developed along the microrough surface of the threads and pitch of the screw. In addition, mature lamellar bones were observed on the irregular and micro-rough surface of the screw, and they had a strong relationship with the implanted screw. On the other hand, the tissues around the machined screws were the immature bones, such as giant cells, cartilage, and woven bone. Moreover, the mixtures of giant cells and fibroblast surrounding fibrous tissues were formed on the interface between the machined screw and new bone by foreign body reactions. The 3D printed Ti6Al4V screws $(35.9 \pm 10.2 \%)$ showed higher bone-to-implant contact than the machined Ti6Al4V screws $(5.9 \pm 3.8 \%)$. This indicated that the rough and irregular surface structures of the 3D-printed Ti6Al4V implant promoted the ingrowth of bone into the micro-pores of the screw and also the BIC of the 3D-printed Ti6Al4V screw was significantly higher than that of the machined screw.

Conclusions : 3D-printed Ti6Al4V screws with a micro-rough surface provides a more favourable environment than machined Ti6Al4V screws with a smooth surface for osseointegration and bone regeneration around the screw implant.

\section{References}

[1] P. Heinl, L. Müller, C. Körner, R.F. Singer, F.A. Müller, "Cellular Ti-6Al-4V structures with interconnected macro porosity for bone implants fabricated by selective electron beam melting," Acta biomaterialia, vol. 4, no. 5, pp. 15361544, 2008.

[2] F.A. Shah, A. Snis, A. Matic, P. Thomsen, A. Palmquist, "3D printed Ti6Al4V implant surface promotes bone maturation and retains a higher density of less aged osteocytes at the bone-implant interface," Acta biomaterialia, vol. 30, pp. 357-367, 2016. 
[3] K. Li, C. Wang, J. Yan, Q. Zhang, B. Dang, Z. Wang, Y. Yao, K. KLin, Z. Guo, L. Bi, Y. Han, "Evaluation of the osteogenesis and osseointegration of titanium alloys coated with graphene: an in vivo study," Scientific reports, vol. 8, pp. 1-10, 2018. 